\title{
Alcohol assessment and feedback by e-mail for university students: main findings from the AMADEUS-1 randomised controlled trial
}

\author{
Jim McCambridge ${ }^{1 *}$, Marcus Bendtsen ${ }^{2,3}$, Nadine Karlsson², lan R White ${ }^{4}$, Per Nilsen², Preben Bendtsen ${ }^{2}$ \\ From International Network on Brief Interventions for Alcohol and Other Drugs (INEBRIA) Meeting 2013 \\ Rome, Italy. 18-20 September 2013
}

\section{Background}

Brief interventions can be efficacious in changing alcohol consumption and related problems and increasingly take advantage of the internet to reach high risk populations such as students.

\section{Aims}

To evaluate the effectiveness of a brief online intervention, part of the national strategic response in Sweden, controlling for the possible effects of the research process.

\section{Methods}

A three arm parallel groups design permitted exploration of the magnitude of the feedback and assessment component effects via randomisation to fully automated: 1) routine practice assessment and feedback; 2) assessment only without feedback; or 3) no contact and thus neither assessment nor feedback. The study was undertaken simultaneously in two universities randomizing the e-mail addresses of all 14,910 students $(4,969,4969$ and 4972 respectively to Groups 1-3) who were entirely blinded to trial participation. Outcomes were evaluated after 3 months via an invitation to participate in a brief crosssectional lifestyle survey.

\section{Results}

Overall, $52 \%(n=7,809)$ of all students completed follow-up, with small differences in attrition between the three groups $(2,546,2,594$ and 2,669 respectively in Groups 1-3). For each of the two primary outcomes, there was one statistically significant difference between groups, with Group 1 having 3.7\% fewer risky drinkers at follow-up than Group 3 $(\mathrm{P}=0.006)$ and Group 2 scoring 0.16 points lower than Group 3 on the AUDIT-C $(\mathrm{P}=0.039)$.

\section{Conclusions}

This study provides some evidence of population-level benefit attained through intervening with individual students.

\section{Trial registration \\ ISRCTN28328154}

\section{Authors' details}

${ }^{1}$ Faculty of Public Health \& Policy, London School of Hygiene \& Tropical Medicine, UK. ${ }^{2}$ Department of Medicine and Health, Linköping University, Sweden. ${ }^{3}$ Department of Computer and Information Science, Linköping University, Sweden. ${ }^{4} \mathrm{MRC}$ Biostatistics Unit, Institute of Public Health, Cambridge University, UK.

Published: 4 September 2013

doi:10.1186/1940-0640-8-S1-A48

Cite this article as: McCambridge et al:: Alcohol assessment and feedback by e-mail for university students: main findings from the AMADEUS-1 randomised controlled trial. Addiction Science \& Clinical Practice 2013 8(Suppl 1):A48.

\footnotetext{
* Correspondence: Jim.McCambridge@lshtm.ac.uk

${ }^{1}$ Faculty of Public Health \& Policy, London School of Hygiene \& Tropical

Medicine, UK

Full list of author information is available at the end of the article
} 